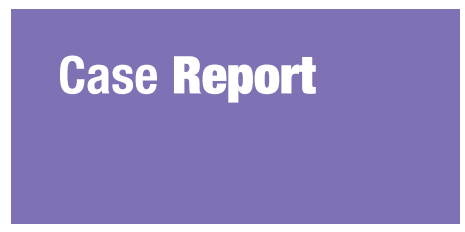

Submitted: 27 May 2015

Accepted: 27 Mar 2016

Online: 7 Dec 2016

\section{Recurrent Intestinal Obstruction in a Patient with Selective IgA Deficiency}

\author{
Chee-Kin HuI ${ }^{1,2}$
}

1 Centre for Alimentary Studies, Endoscopy Centre 1, Lower Albert Road, Central Hong Kong, Hong Kong SAR, China

2 Quality Healthcare Medical Services, 6/F HK Pacific Centre, 28 Hankow Road, Tsim Sha Tsui, Hong Kong, China

To cite this article: Hui C-K. Recurrent intestinal obstruction in a patient with selective IgA deficiency. Malays $J$ Med Sci. 2016;23(6):123-127. http://dx.doi.org/10.21315/mjms2016.23.6.14

To link to this article: http://dx.doi.org/10.21315/mjms2016.23.6.14

\begin{abstract}
A 32 year old woman presented with acute onset of abdominal pain and fever. An urgent computerised tomography (CT) of the whole abdomen showed dilated loop at the terminal ileum in the right lower abdomen with thickening of the wall and oedema. The CT was suggestive of distal small bowel obstruction at the ileum with surrounding wall oedema. Multiple biopsies taken from the terminal ileum and colon on colonoscopy were all unremarkable. She represented oneyear later with a recurrence of intestinal obstruction. CT enteroclysis showed collapse at the distal $3 \mathrm{~cm}$ segment of the terminal ileum. There was no associated wall thickening, active inflammatory changes or ileitis. This was suspicious of post-inflammatory change or fibrosis. She was subsequently found to have selective IgA deficiency with recurrent infection in the terminal ileum resulting in intestinal obstruction. In conclusion, selective IgA deficiency should be considered in patients with recurrent intestinal obstruction without anatomical obstructions.
\end{abstract}

Keywords: selective IgA deficiency, intestinal obstruction, antibiotic prophylaxis

\section{Case History}

A 32-year old woman presented with a oneday history of acute abdominal pain, fever, chills and rigors. She had been suffering from loose stools three times a day with occasional mucous mixed with stool for one month before the acute onset of abdominal pain and fever. She had no travel history for the last six months and was not taking any medication. She had a history of good health and was not at risk for any communicable diseases. She had not been sexually active in the last seven months. There were no significant diseases in her family.

Physical examination showed a tender abdomen with no rebound or rigidity and no clinical signs of pelvic inflammatory disease on gynaecological examination.

Her white cell count was raised to $22 \times 10^{9} / \mathrm{L}$ (normal range 4-11) with 90\% neutrophil, erythrocyte sedimentation rate
$73 \mathrm{~mm} / \mathrm{hr}$ (normal range < 35) and C-reactive protein $98 \mathrm{mg} / \mathrm{L}$ (normal range < 10). Computerised tomography (CT) of the whole abdomen showed a dilated loop of ileum in the right lower abdomen with wall thickening and oedema. There was also distension of the proximal small bowel with fluid seen suggestive of intestinal obstruction (IO).

Chest X-ray, stool culture, for ova and parasites, mid-stream urine culture, blood culture, anti-amoebic antibody and Widal test was all negative. Endocervical swab for bacterial and fungal culture were negative. Screenings for sexually transmissible diseases using nucleic acid amplification tests for Chlamydia trachomatis and Neisseria gonorrhoea were negative. Human immunodeficiency virus I and II antibodies and serology for syphilis were also negative.

Investigation for tuberculous infection with Quantiferon Tuberculous Gold Test, stool for acid fast bacilli smear, stool for Mycobacteria 
tuberculosis culture, stool for Mycobacteria tuberculosis polymerase chain reaction, early morning urine for acid fast bacilli smear and early morning urine for Mycobacteria tuberculosis culture were all unremarkable.

She was treated conservatively and was put on intravenous meropenem and amikacin. The antibiotics were empirically started as there was evidence of infection with fever, chills, rigors, loose stools and raised infective markers. Her intestinal obstruction resolved after three days of conservative management. A CT of the whole abdomen repeated five days later showed resolution of the small bowel obstruction. The wall thickening and oedema of the ileum had also resolved completely.

Upper endoscopy, colonoscopy and small bowel capsule endoscopy were performed upon resolution of the IO. The upper endoscopy was normal down to the second part of the duodenum. The colonoscopy was passed to $25 \mathrm{~cm}$ beyond the ileocecal valve. Multiple lymphoid follicles were seen in the terminal ileum. The colonic mucosa was unremarkable. The capsule endoscopy was unremarkable except for multiple lymphoid follicles in the terminal ileum.

Blind biopsies taken from the terminal ileum showed reactive lymphoid aggregates in the lamina propria. Terminal ileal biopsies for acid fast bacilli smear, Mycobacteria tuberculosis culture and Mycobacteria tuberculosis polymerase chain reaction were all negative.

Biopsies from the duodenum and colon were all unremarkable. Anti-saccharomyces cerevisiae antibody (ASCA), anti-neutrophil cytoplasmic antibody, stool for lactoferrin and stool for calprotectin were performed after the IO had resolved in order to search for evidence of Crohn's disease. They were all negative.

She remained asymptomatic until 11 months later, when she re-presented with fever and acute abdominal pain. Once again the abdominal pain and fever was preceded by a three week history of loose stool three to four times a day, with occasional mucous mixed with the stool.

Her white cell count was raised to $26 \times 10^{9} / \mathrm{L}$ (normal range 4-11) with $88 \%$ neutrophil, erythrocyte sedimentation rate $67 \mathrm{~mm} / \mathrm{hr}$ (normal range < 35) and C-reactive protein $78 \mathrm{mg} / \mathrm{L}$ (normal range < 10). Abdominal $\mathrm{X}$-ray showed multiple fluid levels suggestive of IO. She was again treated conservatively with intravenous meropenem and amikacin. The IO resolved after four days.
In order to exclude the possibility of a false negative result at her first presentation, chest X-ray, Quantiferon Tuberculous Gold Test, stool for culture, stool for acid fast bacilli smear, stool for Mycobacteria tuberculosis culture, stool for Mycobacteria tuberculosis polymerase chain reaction, early morning urine for acid fast bacilli smear, early morning urine for Mycobacteria tuberculosis culture, anti-amoebic antibody, Widal test and blood for culture were all repeated. They were again negative.

As she did not have any clinical signs of guarding, rigidity or rebound, and, as her IO resolved after four days, our surgical colleagues decided against a diagnostic laparoscopy at this juncture. In view of her recurrent IO, a CT enteroclysis with the use of a neutral enteral material and intravenous contrast was performed.

CT enteroclysis is a hybrid technique that combines the use of fluoroscopic intubationinfusion and abdominal CT to examine the small bowel. It combines the advantages of a CT scan and a barium enteroclysis. CT enteroclysis is more sensitive and accurate for the detection of small bowel pathology, and, can complement small bowel capsule endoscopy in the investigation of small bowel pathology (1).

The CT enteroclysis showed collapse at the distal $3 \mathrm{~cm}$ segment of the terminal ileum. There was no associated wall thickening, active inflammatory changes or ileitis. This was suspicious of post-inflammatory changes or fibrosis (Figure 1A and Figure 1B). Several shotty lymph nodes less than $0.4 \mathrm{~cm}$ in size were seen in the right lower quadrant. These lymph nodes appeared reactive in nature. There were no other enlarged lymph nodes found in the other parts of the abdomen or pelvis. Furthermore, no ascites or intra-abdominal fluid collection was detected on CT enteroclysis.

A colonoscopy was repeated and the colonoscope was passed to $30 \mathrm{~cm}$ beyond the ileocecal valve. Multiple lymphoid follicles were seen in the terminal ileum. Biopsies of the terminal ileum at the distal $3 \mathrm{~cm}$ of the terminal ileum and proximal to the lesion seen on CT enteroclysis showed reactive lymphoid aggregates. Terminal ileal biopsies for acid fast bacilli smear, Mycobacteria tuberculosis culture and Mycobacteria tuberculosis polymerase chain reaction were once again negative. As the colonoscope could be passed easily to $30 \mathrm{~cm}$ beyond the ileocecal valve (beyond the $3 \mathrm{~cm}$ pathology seen in the terminal ileum on CT enteroclysis), the segment seen on CT 


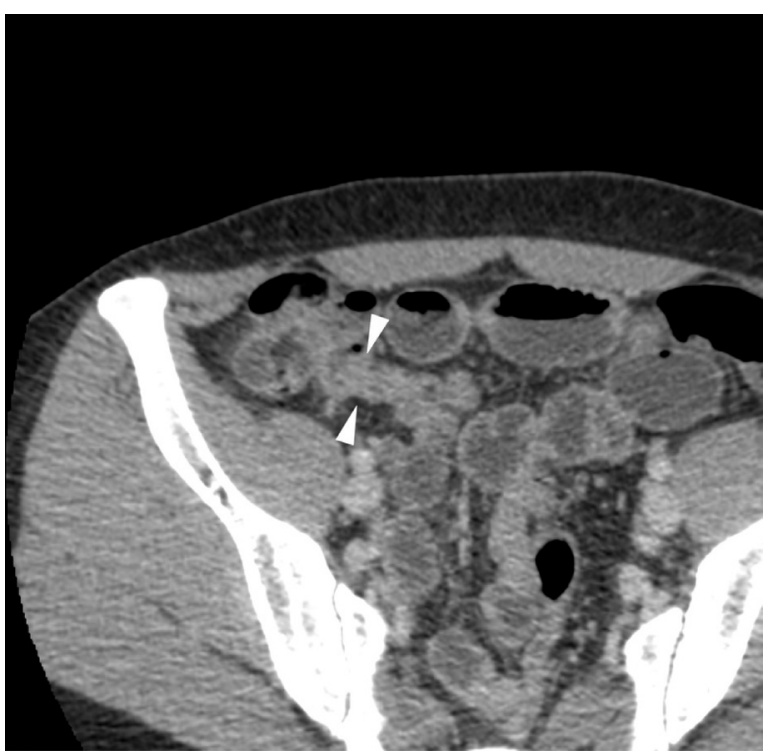

(A)

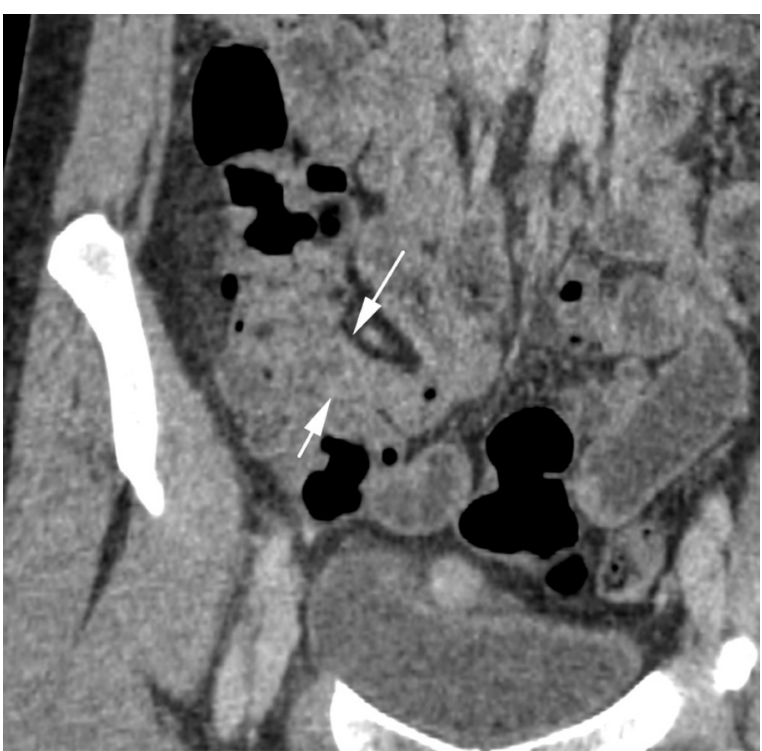

(B)

Figure 1. (A) Axial view of computerised tomography with enterolysis showing persistent collapse at the distal $3 \mathrm{~cm}$ segment of the terminal ileum suspicious of post-inflammatory changes or fibrosis; and (B) coronal view of computerised tomography with enterolysis showing persistent collapse at the distal $3 \mathrm{~cm}$ segment of the terminal ileum suspicious of postinflammatory changes or fibrosis.

enteroclysis was probably a collapse rather than a stenosis.

As both episodes of IO were preceded by loose stool, mucous mixed with stool, increased frequency of bowel motion, raised erythrocyte sedimentation rate and C-reactive protein, a search for a possible inflammatory or infective cause showed low serum immunoglobulin (Ig) A $0.02 \mathrm{~g} / \mathrm{L}$ with normal IgG and IgM.

All other autoimmune markers, IgG subclasses and complement levels were unremarkable. Her ASCA, stool for lactoferrin and stool for calprotectin performed one month after the second IO had resolved, were again unremarkable.

She was diagnosed with selective IgA deficiency (SIgAD) complicated by recurrent infection at the terminal ileum, resulting in IO.
She was put on prophylactic levofloxacin 500 mg daily. She did not have another episode of IO on follow-up for 30 months after levofloxacin prophylaxis. Serial serum IgA remained lower than $0.07 \mathrm{~g} / \mathrm{L}$ with normal $\mathrm{IgG}$ and IgM on regular follow-up for 30 months postlevofloxacin prophylaxis (Table 1).

However, she developed bilateral pitting ankle oedema and frothy urine 24 months after commencement of levofloxacin prophylaxis. Her 24-hour urine showed an albumin of $5.3 \mathrm{~g} /$ day. Anti-nuclear factor antibody, antidouble stranded DNA antibodies, antibodies to Extractable Nuclear Antigens, serum free light chains, cryoglobulins, hepatitis B surface antigen and hepatitis $\mathrm{C}$ virus antibody were all negative.

She declined a renal biopsy for investigation of her nephrotic syndrome at her last follow-up.

Table 1. Table showing serial serum immunoglobulin levels. Ig- Immunoglobulin.

\begin{tabular}{lcccccccc} 
Follow-up from commencement of & $\mathbf{0}$ & $\mathbf{3}$ & $\mathbf{6}$ & $\mathbf{9}$ & $\mathbf{1 2}$ & $\mathbf{1 8}$ & $\mathbf{2 4}$ & $\mathbf{3 0}$ \\
levofloxacin prophylaxis (months) & & & & & & & \\
Serum IgA (normal range 0.87-4.74 g/L) & 0.02 & 0.04 & 0.06 & 0.05 & 0.06 & 0.04 & 0.04 & 0.05 \\
Serum IgG (normal range 6.81-16.48 g/L) & 7.77 & 10.00 & 10.75 & 10.11 & 10.23 & 10.71 & 11.11 & 11.00 \\
Serum IgM (normal range 0.48-3.12 g/L) & 0.50 & 0.49 & 0.56 & 0.61 & 0.63 & 0.71 & 0.88 & 0.92 \\
\hline
\end{tabular}




\section{Discussion}

When a pathology or abnormality is found in the terminal ileum, the most common differential diagnoses are tuberculosis, small bowel lymphoma, Yersinia infection, neuroendocrine tumour or inflammatory bowel disease $(2,3,4)$. As conservative measures resulted in a rapid resolution of IO in this patient along with lymphoid aggregates in the terminal ileum on repeated biopsies, an infection was suspected to be the cause of her recurrent IO. Although lymphoid aggregates in the mucosa of the terminal ileum usually have no clinical significance, they can also be a mucosal response to stimuli such as an infection or inflammatory bowel disease (5).

However, the ASCA, stool for calprotectin, stool for lactoferrin, capsule endoscopy, CT enteroclysis and repeated endoscopic biopsies failed to show any evidence of Crohn's disease. Furthermore, work-ups for tuberculous infection involving the gastrointestinal tract, typhoid and amoebiasis were also repeatedly negative.

SIgAD is the most common form of primary immunoglobulin deficiency. SIgAD is defined as a serum IgA level of less than $0.07 \mathrm{~g} / \mathrm{L}$ with normal serum IgM and IgG in children older than four years of age (6). The incidence of SIgAD in the Chinese population has been reported as $1: 2,600$ to $1: 5,300$ (6).

In the majority of individuals with SIgAD, the deficiency is discovered incidentally during blood donation $(6,7,8)$. A minority of people with SIgAD are likely to suffer from recurrent sinopulmonary infections, allergic diseases, Celiac disease or autoimmune disorder (7), so it is not surprising that the patient had no previous episodes of recurrent infection.

However, SIgAD has not been found to be associated with an increased risk of infection in the gastrointestinal system (8). This is, then, the first case report of SIgAD presenting with recurrent IO due to recurrent infection at the terminal ileum.

I postulate that infection in the terminal ileum may have caused inflammation of the mucosa which may have caused oedema and wall thickening leading to the occurrence of IO. Another possible hypothesis is that mucosal inflammation may have impaired smooth muscle contractility and decreased the release of neurotransmitters (5), causing impaired motor abnormalities in the terminal ileum and IO (5). Repeated infection at this site may have caused the formation of fibrous and granulation tissues in the terminal ileum accounting for the abnormality seen on CT enterolysis.

In view of her recurrent IO and infection at the terminal ileum, the patient was empirically commenced on antibiotic prophylaxis. Although there is no data on the use of antibiotic prophylaxis in the prevention of recurrent gastrointestinal infections in patients with SIgAD it seemed to be effective, as she remained asymptomatic during the follow-up period.

In conclusion, SIgAD should be considered in patients with recurrent IO without anatomical obstructions. The use of antibiotic prophylaxis to prevent recurrent gastrointestinal infections in patients with SIgAD requires further investigation.

\section{Acknowledgement}

Dr. Kui Fai Chan for the radiological images, Dr. Kian Ah-Chong for the surgical management and Dr. Nises C Poddar for Gynaecological management.

\section{Conflict of Interest}

None

\section{Funds}

None

\section{Correspondence}

Dr. Chee-Kin Hui

MBBS (HKU), MD (HKU)

Consultant.

Block 207, Tao Payoh North, \#03-1241, Singapore

Tel: 85227231183

Fax: 85227236620

E-mail: bckhui@gmail.com

\section{References}

1. Maglinte DD, Sandrasegaran K, Lappas JC, Chiorean M. CT enterolysis. Radiology. 2007;245(3):661-671.

2. Hui CK. Celiac disease presenting as idiopathic terminal ileum obstruction. Journal of Gastroenterology and Hepatology Research. 2013;2(6):652-654. 
3. Hui CK. Lymphocytic colitis complicated by a mass in the terminal ileum. Singapore Med $J$. 2015;56(5):e85-e88. http://dx.doi.org/10.11622/ smedj.2015080.

4. Hui CK. A case of corpus callosum lesion associated with disseminated mycobacterium tuberculosis infection. IIOAB J. 2013;4(2):28-31.

5. Koklu S, Coban S, Ertugrul I, Basar O, Ensari A, Akyol A, et. al. Intestinal obstruction in Celiac disease: case report. Dig Dis Sci. 2004;49(9):1485-1458.

6. Yel L. Selective IgA deficiency. $J$ Clin Immunol. 2010;30(1):10-16. http://dx.doi.org/10.1007/ s10875-009-9357-X.
7. Singh K, Chang C, Gershwin ME. IgA deficiency and autoimmunity. Autoimmun Rev. 2014;13(2):163-177. http://dx.doi.org/10.1016/j. autrev.2013.10.005.

8. Jorgensen GH, Gardulf A, Sigurdsson MI, Sigurdardottir ST, Thorsteinsdottir I, Gudmundsson S, et. al. Clinical symptoms in adults with selective IgA deficiency: a case-control study. J Clin Immunol. 2013;33(4):742-747. http://dx.doi.org/10.1007/s10875-012-9858-x. 